\title{
Sur le plus grand commun diviseur de plusieurs nombres.
}

(Par Ernest Ces̀̀ro, étudiant, à Torre Annunziata.)

1. Soit $\left(x_{1}, x_{2}, x_{3}, \ldots, x_{m}\right)$ le plus grand commun diviseur des nombres $x_{1}, x_{2}, \ldots, x_{m}$. Pour évaluer la somme de toutes les quantités, analogues à $F\left(x_{1}, x_{2}, \ldots, x_{m}\right)$, lorsque chaque $x$ varie, indépendamment des autres, depuis 1 jusqu'à $n$, on pourrait ordonner le résultat par rapport à $F(1), F(2), F(3), \ldots$, en cherchant combien de groupes de $m$ nombres, égaux ou inégaux, non supérieurs $\grave{a} n$, admettent $p$ pour plus grand commun diviseur. Mais il est plus facile d'imaginer une fonction $f$, telle que l'on ait

$$
f(a)+f(b)+f(c)+\cdots=F(x)
$$

$a, b, c, \ldots$ étant tous les diviseurs de $x$, et d'ordonner la somme considérée par rapport à $f(1), f(2), f(3), \ldots$ En effet, le coefficient de $f(p)$ exprimera combien de groupes de m nombres, égaux ou inégaux, non supérieurs à n, admettent $p$ pour diviseur commun, que celui-ci soit ou non le plus grand. Or, puisque chaque $x$ n'est susceptible que des valeurs $p, 2 p, 3 p, \ldots, q_{p} p$, le coefficient cherché est $q_{p}^{m}$. Il en résulte

$$
\Sigma F\left(x_{1}, x_{2}, x_{3}, \ldots, x_{m}\right)=q_{1}^{m} f(1)+q_{2}^{m} f(2)+q_{3}^{m} f(3)+\cdots
$$

$\grave{\mathbf{A}}$ cette formule on peut $\left(^{*}\right)$ substituer la suivante:

$$
\Sigma F\left(x_{1}, x_{2}, x_{3}, \ldots, x_{m}\right)=\psi\left(q_{1}\right)+\left(2^{m}-1\right) \psi\left(q_{2}\right)+\left(3^{m}-2^{m}\right) \psi\left(q_{3}\right)+\cdots,
$$

pourru que l'on pose

$$
\psi(x)=f(1)+f(2)+f(3)+\cdots+f(x) .
$$

Les formules (2) et (3) sont fort utiles dans les recherches asymptotiques, re- 
latives au plus grand commun diviseur de $m$ nombres. Mais l'on verra plus loin pourquoi nous ne nous $\mathrm{y}$ arrêtons pas.

2. On peut écrire

$$
q_{p}^{m}=\frac{n^{m}}{p^{m}}-\varepsilon m \frac{n^{m-1}}{p^{m-1}},
$$

$\varepsilon$ étant une fraction proprement dite. Le second membre de (2), divisé par $n^{m}$, prend donc la forme

$$
\sum_{p=1}^{p=n} \frac{f(p)}{p^{m}}-\frac{\varepsilon m}{n} \sum_{p=1}^{p=n} \frac{f(p)}{p^{m-1}}
$$

Si la fonction $f(x)$ reste entre deux limites finies, lorsque $x$ varie de 1 à $+\infty$, il est clair que la somme $\sum_{\mathbf{i}}^{n} \frac{f(p)}{p^{r}}$ n'est pas d'un ordre supérieur à celui de $\sum_{i}^{n} \frac{1}{p^{r}}$. Lors, donc, que $r$ est supérieur à l'unité, la somme en question est de l'ordre des constantes, au moins. Dans le cas de $r=1$, elle n'est pas d'un ordre supérieur à celui de $\log n$, et son rapport à $n$ tend, par conséquent, vers zéro. Il en résulte que, pour $n$ indéfiniment croissant, l'expression (4) tend vers

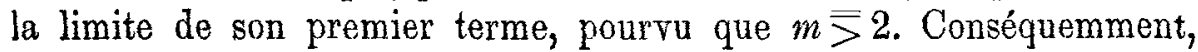

$$
\lim \cdot \frac{1}{n^{m}} \mathbf{\Sigma} F\left(x_{1}, x_{2}, x_{3}, \ldots, x_{m}\right)=\sum_{p=1}^{p=\infty} \frac{f(p)}{p^{m}} .
$$

3. Pour appliquer la formule (5), il faudrait chercher, chaque fois, la fonction $f$, qui dépend de la fonction donnée $F$ par la relation (1); mais nous pourons nous affranchir de cela, en observant que, d'après les principes exposés dans le Premier Mémoire, l'identité (1) donne lieu à la formule

Par suite:

$$
\sum_{p=1}^{p=\infty} \frac{1}{p^{m}} \cdot \sum_{p=1}^{p=\infty} \frac{f(p)}{p^{m}}=\sum_{p=1}^{p=\infty} \frac{F(p)}{p^{m}} .
$$

$$
\lim \cdot \frac{1}{n^{m}} \Sigma F\left(x_{1}, x_{2}, x_{3}, \ldots, x_{m}\right)=\frac{1}{s_{m}} \sum_{p=1}^{p=\infty} \frac{F(p)}{p^{m}} .
$$

4. La formule (6) est d'une extrême souplesse pour la résolution des questions de probabilités, concernant le plus grand commun diviseur de $m$ nombres, pris au hasard. Veut-on savoir, par exemple, quelle probabilité il y a pour que ce diviseur soit précisément un nombre donné $k$ ? Faisons $F(x)=1$, 
pour $x=k$, mais $F(x)=0$, en général. La formule (6) donne immédiatement

$$
P_{k}=\frac{1}{k^{m} s_{m}}
$$

En particulier, pour $k=1$ et $m=3$ :

"La probabilité que trois nombres, pris au hasard, soient premiers entre eux, est $0,83190 \ldots "$, etc., etc....

5. Si nous donnions d'autres applications de la formule (6), cette Note ferait, en quelque sorte, double emploi avec une des Notes qui précèdent. En effet, d'après la formule (19) de notre article "Le plus grand diviseur carre" on a:

$\lim \cdot \frac{1}{n^{m}} \cdot \Sigma F\left(x_{1}, x_{2}, x_{3}, \ldots, x_{m}\right)=\lim \cdot \frac{F\left(d_{m}^{\frac{1}{m}}(1)\right)+F\left(d_{m}^{\frac{1}{m}}(2)\right)+\cdots+F\left(d_{m}^{\frac{1}{m}}(n)\right)}{x_{2}}$.

Par conséquent:

"La probabilité que la me racine de la plus haute puissance mème, qui divise un nombre entier pris au hasard, appartienne à un certain système de nombres, ne diffère pas de la probabilité que le plus grand commun diviseur de $m$ entiers, pris au hasard, appartienne au même système".

Ailleurs $\left({ }^{*}\right)$, nous avons donné l'explication, extrêmement simple, de ce fait curieux.

6. Il n'y aurait done à faire autre chose, pour terminer cet article, que de transcrire l'article cité, en remplaçant partout la fonction $\frac{1}{d_{m}^{m}}(x)$ par $\left(x_{1}\right.$, $\left.x_{2}, \ldots, x_{m}\right)$. Aussi, nous bornerons-nous à énoncer quelques propositions:

1. "La probabilité que le plus grand commun diviseur de $m$ nombres, pris au hasard, soit un carré, est $\frac{s_{2 m}}{s_{m}} n$.

2. " Il y a 7 à parier, contre 3 , que le plus grand commun diviseur de deux nombres, pris au hasard, est composé d'un nombre pair, plutôt que d'un nomóre impair de facteurs premiers, égaux ou inégaux".

3. "Il est trois fois plus probable que le plus grand commun diviseur de deux entiers, pris au hasard, soit impair plutôt que pair ".

$4 .^{\circ}$ a) Il y a 18 à parier, contre 7 , que le plus grand commun diviseur de deux entiers, pris au hasard, se termine par un chiffre impair, autre que 5. 
b) Il y a 3 à parier, contre 97, qu'il se termine par 5; et 1 seulement, contre 99 , qu'il se termine par 0 .

différent de 0 .

c) Il y a 6 à parier, contre 19, qu'il se termine par un chiffire pair,

5. ${ }^{\circ}$ On a, en moyenne,

$$
\begin{array}{ll}
\lambda(x, y)=\frac{2}{5}, & \lambda(x, y) \mu(x, y)=\frac{90}{\pi^{4}}, \\
\mu(x, y)=\frac{36}{\pi^{4}}, & \mu(x, y) \mathfrak{e}\{(x, y) \varepsilon\}=\frac{24}{\pi^{4}} \sin ^{4} \pi \varepsilon,
\end{array}
$$

etc., etc....

(') Voyez Premier Mémoire d'Arithmétique.

(“) Mathesis, 1884: Probabilité de certains faits arithmétiques. 